\title{
The role of Active Galactic Nuclei feedback in the formation of the brightest cluster galaxies
}

\author{
Davide Martizzi ${ }^{1}$, Romain Teyssier ${ }^{1,2}$ and Ben Moore ${ }^{1}$ \\ ${ }^{1}$ Institute for Theoretical Physics, University of Zurich, CH-8057 Zürich, Switzerland \\ email: martdav@physik.uzh.ch, teyssier@physik.uzh.ch, moore@physik.uzh.ch \\ ${ }^{2}$ CEA Saclay, DSM/IRFU/SAP, Bâtiment 709, F-91191 Gif-sur-Yvette, Cedex, France
}

\begin{abstract}
The formation of the brightest cluster galaxies (BCG) is a challenge for galaxy formation theory. We performed high resolution cosmological hydrodynamical simulations with the AMR code RAMSES to study the properties of the BCG which forms at the center of a Virgo-like cluster. We compare the results of 2 galaxy formation scenarios, one in which only supernovae feedback is included, and one in which also AGN feedback is considered. Properties of the simulated BCG which are comparable with those of observed massive elliptical galaxies and BCGs cannot be obtained if AGN feedback is not considered. The stellar-to-halo mass ratio in simulations without AGN feedback appears too large when compared to observations, while it is compatible the observationally determined values when AGN feedback is included. The kinematical and structural properties of the BCG are extremely different in the two models. When we do not include AGN feedback, the BCG is quickly rotating, with high Sérsic index, a clear mass excess in the center and a very large stellar mass fraction. When AGN feedback is considered, the BCG is slowly rotating, with a significantly cored surface density profile and low stellar mass fraction.
\end{abstract}

Keywords. Galaxy Formation - Theory, Galaxy Clusters, Black Holes, Cosmology, Numerical Methods

\section{Introduction}

Galaxy formation models that not include AGN feedback are known to overpredict the masses and star formation rates of BCGs at redshift $z=0$. AGN are expected to provide enough gas heating to prevent excessive star formation, therefore the inclusion of AGN feedback in theoretical models is extremely important when trying to reproduce the properties of BCGs. We use the AMR code RAMSES (Teyssier 2002) to perform two hydrodynamical cosmological simulations of a Virgo-like cluster of galaxies with a virial mass of $M_{\mathrm{vir}}=10^{14} \mathrm{M}_{\odot}$ in the context of the $\Lambda$ CDM cosmological scenario. We achieve a mass resolution of $8.2 \times 10^{6} \mathrm{M}_{\odot}$ and a spatial resolution of $\sim 500 \mathrm{pc}$, that allow us to resolve the formation and evolution of the brightest central galaxy in the cluster. The simulations include gas dynamics and standard galaxy formation recipes, including gas cooling, the effect of UV background, star formation and supernovae feedback. In one run, labeled as AGN-ON, we include AGN feedback adopting a modified version of the Booth \& Schaye (2009) model. In the second simulation we do not include AGN feedback, therefore we labeled it as AGN-OFF. Comparison between the two runs and observational results allowed us to study the relevance of AGN feedback in shaping the 

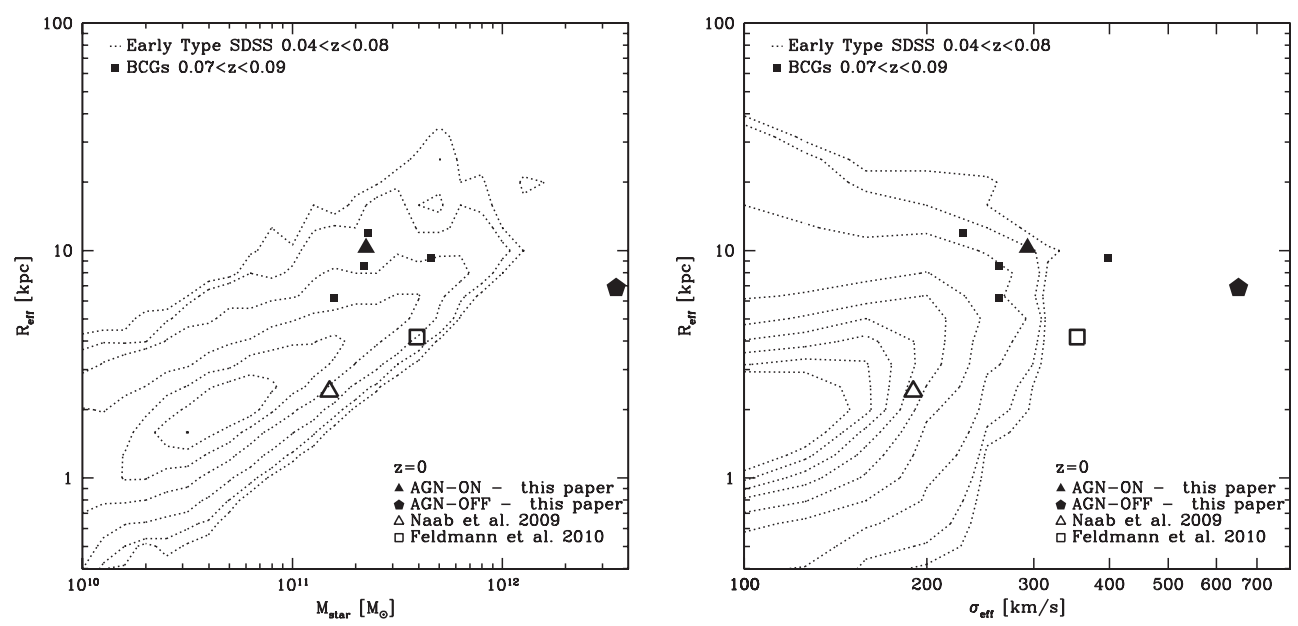

Figure 1. Mass-size (left) and velocity dispersion-size (right) relation of early-type galaxies at redshift $z=0$ from SDSS data (van der Wel et al. 2008), compared to four early-type galaxies from different cosmological simulations. The black dotted lines are contours of the number of early-type galaxies per bin in the $0.04<z<0.08$ sample; going from outside-in we show contours for $5,10,30,100,200,300,400,500,600$ galaxies per bin. Each bin has a size $\Delta \log \left(M_{\text {star }}\right)=\Delta \log \left(R_{\text {eff }}\right)=\Delta \log \left(\sigma_{\text {eff }}\right)=0.1$. The four BCGs analysed by Brough et al. $(2011)$ are also shown as black filled squares. Plot taken from Martizzi et al. (2012).

properties of BCGs. Further details about our simulations can be found in Martizzi et al. (2012).

\section{Results}

We analyse the masses, effective radii and mean velocity dispersions within the effective radius of the BCGs in our models. A comparison is made with the simulations of Naab et al. (2009) and Feldmann et al. (2010) which reproduce some of the observed properties of field elliptical galaxies and central galaxies in groups without AGN feedback. We also compare our model to a sample of elliptical galaxies at $0.04<z<0.08$ (van der Wel et al. 2008) and to four BCGs at $0.07<z<0.09$ (Brough et al. 2011). Figure 1 shows the mass-size and velocity dispersion-size of early-type galaxies and BCGs at low redshift compared to the result of our two simulations and to the results of Naab et al. (2009) and Feldmann et al. (2010). Our results show that the inclusion of AGN feedback helps in bringing the stellar mass, velocity dispersion and size close to those of observed galaxies. In Figure 2 we show the stellar mass vs. halo mass relation predicted by abundance matching (Moster et al. 2010) and we compare it to our simulations. The AGN-OFF model produces a stellar mass that is $\sim 10$ times the expected value, whereas the AGN-ON model predicts a mass that well matches the abundance matching constraints.

We have also analysed the kinematic and structural properties of the simulated BCGs. When we do not include AGN feedback, the BCG is quickly rotating. When AGN feedback is considered, the BCG is slowly rotating. Figure 3 shows the stellar mass surface density profiles compared to Sérsic fits. The AGN-OFF BCG has a high Sérsic index and a mass excess with respect to the fit in the center, a product of a long sequence of wet mergers followed by intense star formation activity. The AGN-ON BCG has a significantly cored surface density profile (very shallow inner slope), a mass deficit with 


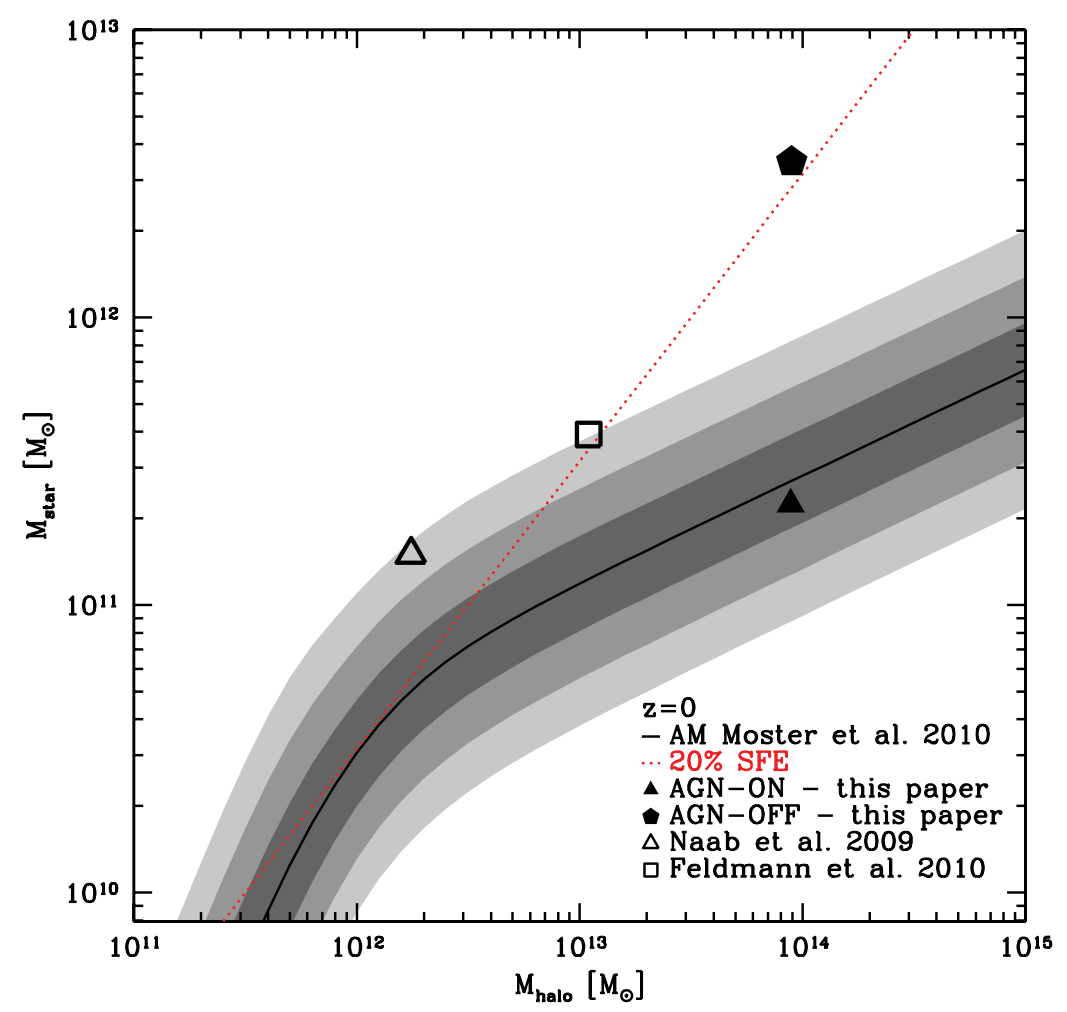

Figure 2. Comparison of the stellar-vs-halo mass relation in 4 early-type galaxies from different cosmological simulations (filled and empty black dots). The dotted line is the relation expected for a $20 \%$ star formation efficiency from the universal baryon fraction. The solid black line is the prediction from abundance matching (Moster et al. 2010). The grey shaded areas represent the $1 \sigma, 2 \sigma$ and $3 \sigma$ scatter bars around the average relation. Plot taken from Martizzi et al. (2012).

respect to the Sérsic fit. In Martizzi et al. (2012b) we have shown that the formation of such a core is related to repeated, AGN driven impulsive gas motions which modify the gravitational potential in the central region of the cluster and generate irreversible modifications in the dark matter and stellar mass distribution. Further results concerning our BCG simulations and their interpretation are discussed in detail in Martizzi et al. (2012).

\section{Conclusions and Future Work}

The analysis of our two simulations and their comparison with observational results lead us to conclude that AGN feedback is an extremely relevant process which deeply influences the evolution of the most massive galaxies in clusters. This effect cannot be neglected when one is interested in modeling the BCGs. We are currently building a catalog of $\sim 100$ cosmological simulations of clusters of galaxies performed with RAMSES and including AGN feedback. The aim of this project is to study the properties of a large number of simulated clusters with different masses and histories, then compare the results to observations and realise constraints on theoretical models. 


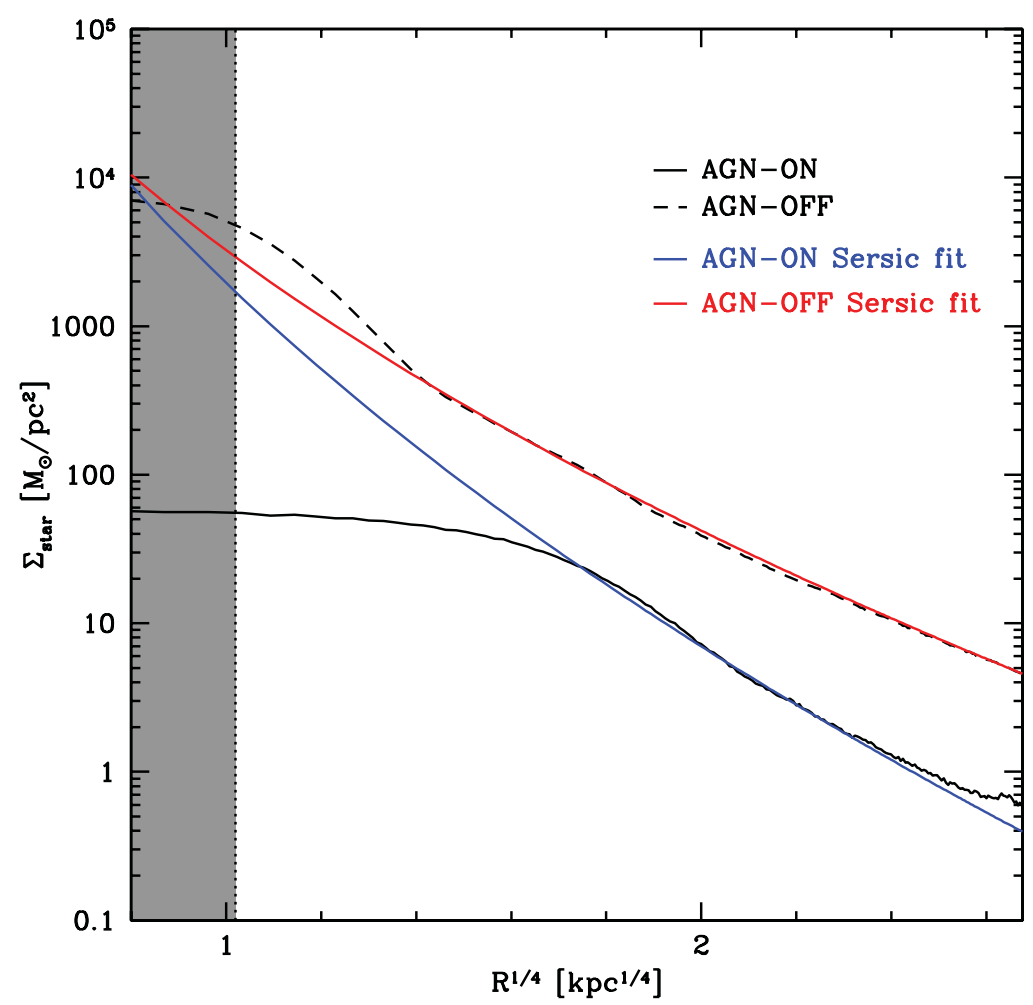

Figure 3. Stellar mass surface density profile of the BCG in our two models, compared with Sersic profiles (see text for details). The black solid lines are for the AGN-ON model and the black dashed lines are for the AGN-OFF model. In both panels the grey shaded area shows the unresolved region of our simulations. Plot taken from Martizzi et al. (2012).

\section{References}

Teyssier, R. 2002, A\& A, 385, 337

Booth, C. M. \& Schaye, J. 2009, MNRAS, 398, 53

Naab, T., Johansson, P. H., \& Ostriker, J. P. 2009, ApJ, 699, L178

Feldmann, R., Carollo, C. M., Mayer, L., et al. 2010, ApJ, 709, 218

Martizzi, D., Teyssier, R., \& Moore, B. 2012, MNRAS, 420, 2859

Moster, B. P., Somerville, R. S., Maulbetsch, C., et al. 2010, ApJ, 710, 903

Brough, S., Tran, K.-V., Sharp, R. G., von der Linden, A., \& Couch, W. J. 2011, MNRAS, 414, L80

van der Wel, A., Holden, B. P., Zirm, A. W., et al. 2008, ApJ, 688, 48

Martizzi, D., Teyssier, R., Moore, B., \& Wentz, T. 2012, MNRAS, 422, 3081 